\title{
Mesure de la concentration absolue de SiO par spectroscopie d'absorption UV
}

\author{
F. Coursimault, O. Motret, R. Viladrosa et J.M. Pouvesle
}

GREMI, UMR 6606, Université d'Orléans, BP. 6759, 45067 Orléans cedex 2, France

\begin{abstract}
Résumé. Le but de cette étude est de développer un diagnostic de mesure de concentration de SiO adapté à un procédé industriel de dépôt d'oxydes de silicium sur polymères par décharge à barrière diélectrique atmosphérique (DBD). Deux méthodes spectroscopiques basées sur des techniques d'absorption et d'auto-absorption ont été développées. La concentration de $\mathrm{SiO}$ a été estimée par ajustement des spectres synthétiques calculés sur les spectres expérimentaux. Ces deux méthodes permettent de suivre l'évolution temporelle de SiO durant les phases de décharge et de post-décharge.
\end{abstract}

\section{INTRODUCTION}

Cette étude ce situe dans le domaine du traitement de surface de films polymères par dépôt d'oxydes de silicium. La connaissance de concentrations absolues d'espèces actives comme $\mathrm{SiO}_{\mathrm{x}}, \mathrm{SiH}_{\mathrm{x}}$, durant la phase plasma représente un paramètre essentiel dans la connaissance et l'optimisation du procédé industriel de dépôt d'oxydes. Parmi les diagnostics optiques développés pour la mesure de concentrations d'espèces, la fluorescence induite par laser apparaît comme la technique la plus pertinente [1-3], mais cette méthode ne semble pas être adaptée à un environnement industriel et peut représenter des coûts d'investissement et de fonctionnement non négligeables. Cette présente étude a été motivée par la mesure de la concentration absolue résolue en temps de SiO par absorption résonante ou par auto-absorption dans un plasma atmosphérique non thermique. Pour de fortes concentrations en réactants l'auto-absorption longitudinale est non négligeable et permet d'estimer la concentration absolue de SiO durant la période de décharge à partir de l'ajustement du spectre synthétique auto-absorbé sur le spectre expérimental. Cette méthode est très intéressante car elle requière uniquement la mise en ouvre du réacteur industriel et nécessite seulement l'obtention du spectre expérimental auto-absorbé. L'absorption résonante est plus complexe et nécessite l'utilisation de deux réacteurs : un comme source de fluorescence de $\mathrm{SiO}(\mathrm{A}-\mathrm{X})$ et l'autre en tant que milieu à sonder. Par contre ce dernier diagnostic présente l'avantage de pouvoir estimer la concentration de $\mathrm{SiO}$ durant toute la période de post-décharge. Le propos de ce travail est de réaliser un diagnostic in-situ compatible avec les contraintes industrielles. Nous présentons dans cet article les résultats spécifiques aux transitions vibrationnelles $\operatorname{SiO}\left(A^{l} I I-X^{l} \Sigma^{+}\right)-\left(v^{\prime}, v^{\prime \prime}\right)=(3,2)$ et $(0,0)$ (têtes de bande 234.3 et $234.5 \mathrm{~nm}$ resp.).

\section{MONTAGE EXPERIMENTAL}

Les molécules de d'oxyde de silicium sont produites dans un réacteur à barrière diélectrique linéaire (lame/plan) à partir de mélanges composés de silane, d'un gaz oxydant et d'argon à la pression atmosphérique. La formation de poudres de $\mathrm{SiO}_{x}$ est limitée grâce la géométrie adaptée du réacteur. Deux réacteurs DBD ont été utilisés pour les mesures d'absorption résonnante : un réacteur sonde et un réacteur «maquette » simulant le réacteur industriel. Ces deux réacteurs ont des géométries similaires $(0.15 \mathrm{~m}$ de longueur), voir figure 1. Pour les mesures en auto-absorption un seul réacteur a été utilisé (le réacteur maquette). Ces réacteurs sont alimentés par des générateurs haute tension impulsionnels (fréquence : 10 $\mathrm{Hz}$, tension appliquée : $30 \mathrm{kV}$ ). Ce type de générateur est capable de produire des tension possédant des fronts de montée très raides $d \mathrm{~V} / \mathrm{d} t>10^{12} \mathrm{~V} / \mathrm{s}$. Pour les mesures basées sur l'absorption la synchronisation entre les deux réacteurs est assurée par un système de déclenchement variable référencé par rapport au 
réacteur maquette. Ce système nous permet d'estimer la concentration de l'espèce moléculaire durant la post-décharge du réacteur maquette. Les spectres de SiO ont été observés avec un spectromètre VUV-UV ARC, $0.50 \mathrm{~m}$ de focale), équipé d'un réseau $3600 \mathrm{tr} / \mathrm{mm}$. Un photomultiplicateur VUV-UV (Hamamatsu R1220) est placé en sortie du spectromètre. Les signaux issus du PM sont analysé au moyen d'un oscilloscope rapide (Tektronix 520A, $500 \mathrm{MHz}$ ) connecté à un PC.

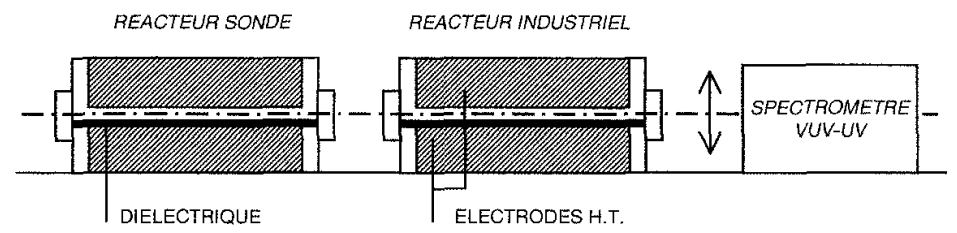

Figure 1. Schéma partiel de l'installation expérimentale

\section{CALCUL DE SPECTRES ROTATIONNELS}

\subsection{Spectres absorbés}

L'intensité d'un spectre ro-vibrationnel transmise après passage dans un milieu absorbant est donné par la loi de Beer-Lambert,

$$
I a_{n^{\prime \prime}}^{n^{\prime}}(v)=\sum_{v^{\prime}, v^{n}, J^{\prime}, J^{\prime \prime}} I_{n^{\prime \prime} v^{\prime \prime} J^{\prime \prime}}^{n^{\prime} J^{\prime} J^{\prime}} V_{e m}(v) \times \exp \left[-\sum_{v^{\prime}, v^{\prime \prime}, J^{\prime}, J^{\prime \prime}}\left(\frac{\Delta N}{N}\right)_{n^{\prime}, v^{\prime}, J^{\prime}}[\operatorname{SiO}] \sigma_{n^{\prime \prime} v^{\prime \prime \prime} J^{\prime \prime}}^{n^{\prime} J^{\prime \prime}} V_{a b s}(v) l\right]
$$

où $l$ est la longueur d'absorption, $I_{n^{\prime \prime} v^{\prime} v^{\prime \prime}{ }^{\prime \prime}}^{\prime \prime}$ l'intensité totale d'une raie rotationnelle en émission, $(\Delta N / N)_{n^{\prime}, v^{\prime}, J^{\prime}}$ la fraction de la population totale des molécules SiO dans le niveau rotationnel $\left(\mathrm{n}^{\prime}, \mathrm{v}^{\prime}, \mathrm{J}^{\prime}\right)$,

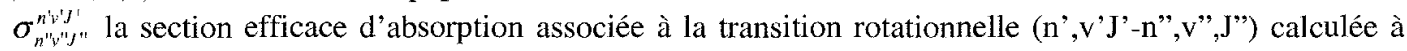
partir de Field et al [6] et $V_{e m}(v)$ et $V_{a b s}(v)$ les profils de Voigt en émission et en absorption respectivement. Avec l'intensité sommée sur toutes les raies contribuant à l'émission à un $v$ donné :

$$
I_{n^{\prime \prime}}^{n^{\prime}}(v)=\sum_{v^{\prime}, v^{\prime \prime}, J^{\prime}, J^{\prime \prime}} I_{n^{\prime} v^{\prime \prime \prime} J^{\prime \prime}}^{n^{\prime} J^{\prime}} V_{e m}(v)
$$

\subsection{Spectres auto-absorbés}

Pour de fortes concentrations en réactants, l'auto-absorption longitudinale ne peut pas être négligée, principalement pour les transitions vibrationnelles $\left(\mathrm{v}^{*}, 0\right)$. L'intensité d'un spectre ro-vibrationnel transmise en sortie d'un milieu auto-absorbant est donné par

$$
I S a_{n^{\prime \prime}}^{n^{\prime}}(v)=I_{n^{\prime \prime}}^{n^{\prime}}(v) \int_{0}^{l} \exp \left[-\sum_{v^{\prime}, v^{\prime \prime}, J^{\prime}, J^{\prime \prime}}\left(\frac{\Delta N}{N}\right)_{n^{\prime}, v^{\prime}, J^{\prime}}[S i O] \sigma_{n^{\prime} v^{\prime \prime} J^{\prime \prime}}^{n^{\prime}, J^{\prime}} V_{a b s,}(v)(l-x)\right] d x
$$

Si la fluorescence auto-absorbée est utilisée comme lumière incidente dans un diagnostic d'absorption le terme $l_{n^{\prime \prime}}^{n^{\prime}}(v)$ doit être remplacé par le terme $I s a_{n^{\prime \prime}}^{n^{\prime}}(v)$ dans l'expression (1).

\section{ESTIMATION DE LA CONCENTRATION ABSOLUE DE SiO}

La connaissance des températures d'excitation (rotationnelle et vibrationnelle) d'un plasma non thermique est très important pour la construction de spectres moléculaires synthétiques. Ces paramètres ont été déterminés dans une étude précédente à partir de transitions vibrationnelles ( $\left.v^{\prime}, v^{\prime \prime} \neq 0\right)$ [7], nous avons obtenu des valeurs moyennes de $\operatorname{Tr}=(1000 \pm 100) \mathrm{K}$ et $\mathrm{TV}=(5000 \pm 300) \mathrm{K}$ respectivement durant la période de fluorescence et pour les conditions expérimentales de cette étude. 
La transition $(3,2)$ dont la tête de bande se situe à $234.3 \mathrm{~nm}$ est très proche de la transition $(0,0)$, c'est pourquoi la transition $(3,2)$ doit être incluse dans la simulation spectrale. La fonction d'appareil $\left(F W H M=\Delta_{a p p}\right)$ a été estimée expérimentalement et utilisée dans la convolution avec les spectres synthétiques avant comparaison avec les spectres expérimentaux.

\subsection{A partir de spectres auto-absorbés}

Pour exemple, la figure $2 a$ présente un spectre intégré durant la période de fluorescence. Le meilleur ajustement (figure 2b) donne une concentration de $\mathrm{SiO}$ de l'ordre de $(4 \pm 1) 10^{14} \mathrm{~cm}^{-3}$, pour les conditions de mélanges (Ar/oxydant $\left./ \mathrm{SiH}_{4}\right)=(98.43 / 1.18 / 0.38)$. Ceci correspond approximativement à $0.44 \%$ de conversion de silane en monoxyde de silicium.

(a)

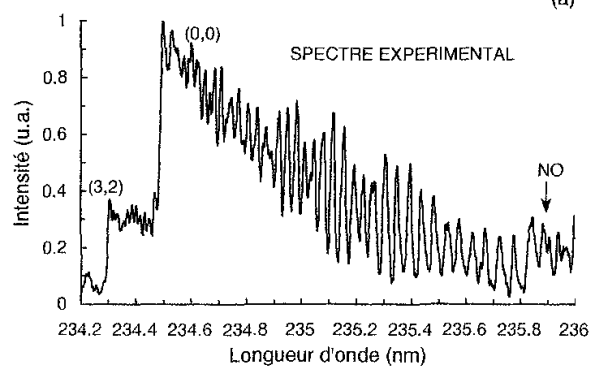

(b)

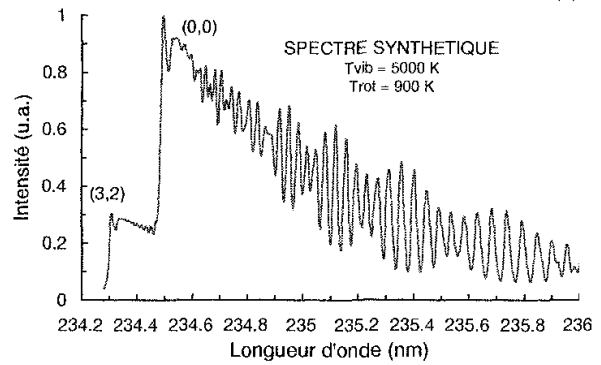

Figure 2. Spectres expérimental (a) et synthétique (b) pour une fluorescence auto-absorbée. $\left(\right.$ Ar/oxydant $\left./ \mathrm{SiH}_{4}\right)=(98.43 / 1.18 / 0.38)-\Delta_{\text {app }}(\mathrm{FWHM})=0.015 \mathrm{~nm}-$ Tension appliquée $=25 \mathrm{kV}$

\subsection{A partir de spectres absorbés}

Pour les mesures d'absorption les deux réacteurs sont utilisés. La première phase consiste à enregistrer le spectre auto-absorbé issu du réacteur sonde et le caractériser par la construction d'un spectre synthétique auto-absorbé. Ensuite ce spectre synthétique est introduit dans la simulation de la lumière absorbée comme lumière incidente pour le réacteur maquette. Figures $3 \mathrm{a}$ et $3 \mathrm{~b}$ montrent un résultat typique obtenu à $\Delta \mathrm{t}=600 \mu \mathrm{s}$ dans la post-décharge du réacteur maquette, pour $\left(\mathrm{Ar} / \mathrm{oxidant} / \mathrm{SiH}_{4}\right)=(99.62 / 0.294 / 0.084)$. Le meilleur ajustement conduit à une concentration $[\mathrm{SiO}]=(1.7 \pm 0.3) 10^{14} \mathrm{~cm}^{-3}$ dans le réacteur maquette.
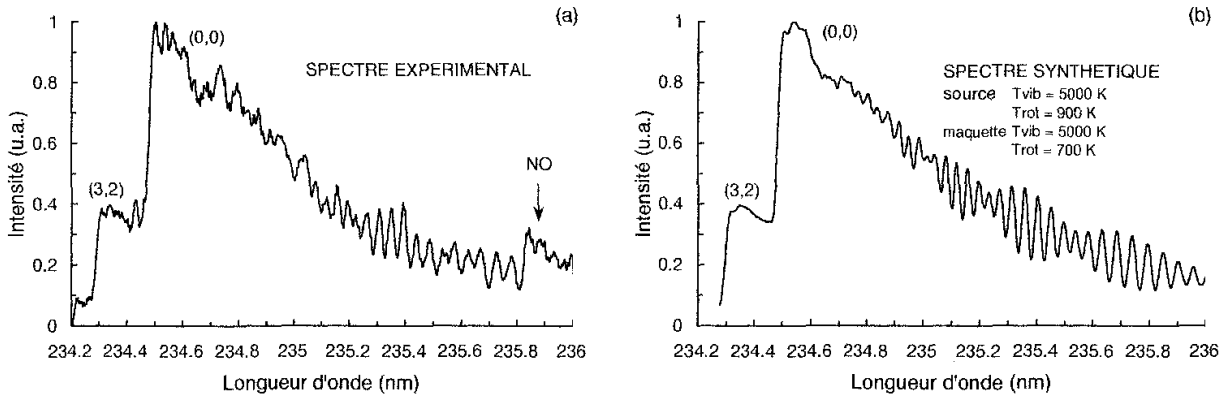

(b)

Figure 3. Spectre expérimental (a) et synthétique (b) pour une fluorescence absorbée.

$\left(\right.$ Ar/oxydant $\left./ \mathrm{SiH}_{4}\right)=(99.62 / 0.294 / 0.084)-\Delta_{\text {app }}(\mathrm{FWHM})=0.025 \mathrm{~nm}-\Delta \mathrm{t}=600 \mu \mathrm{s}-$ Tension appliquée $=18 \mathrm{kV}$

\subsection{Le comportement de SiO}

La figure 4 montre que les molécules de SiO sont formées relativement tard dans la post-décharge et sa durée de vie est très importante. Le point concernant la période de décharge, obtenu par auto-absorption, est en accord avec les autres points. La valeur initiale élevée de la concentration de SiO et sa durée de vie importante laisse supposer un effet cumulatif de la production de $\mathrm{SiO}$ au travers des décharges 
successives. La figure 5 représente la production de $\mathrm{SiO}$ mesurée dans la décharge en fonction de la proportion initiale de silane à rapport oxydant $/ \mathrm{SiH}_{4}$ constant. On constate un phénomène de saturation que nous attribuons à la consommation d'un important précurseur de $\mathrm{SiO}: \mathrm{O}\left({ }^{\mathrm{l}} \mathrm{D}\right)$, par les molécules du gaz oxydant lorsque la concentration de ce dernier devient importante. Ces résultats indiquent qu'il est difficile d'augmenter la concentration de $\mathrm{SiO}$ gazeux dans ces conditions à des valeurs supérieures à environ $5.10^{14} \mathrm{~cm}^{-3}$.

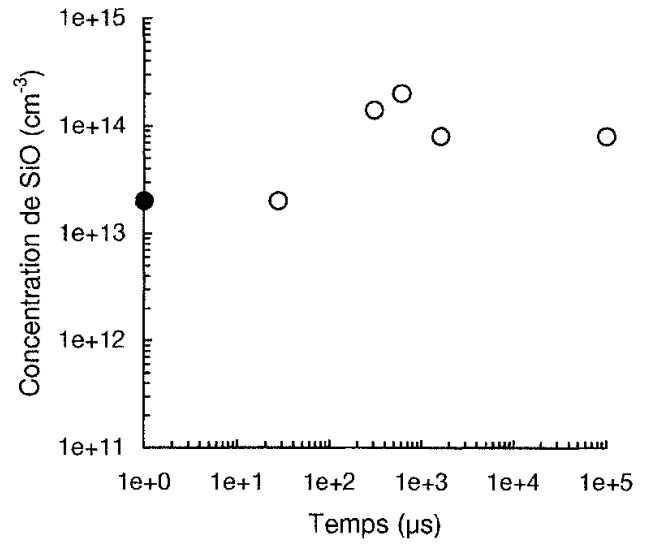

Figure 4. Evolution temporelle de [SiO] déduite de mesures d'auto-absorption $(\bullet$ et absorption (o) - Tension appliquée $=18 \mathrm{kV}$ $\left(\mathrm{Ar} /\right.$ oxydant $\left./ \mathrm{SiH}_{4}\right)=(99.62 / 0.294 / 0.084)$

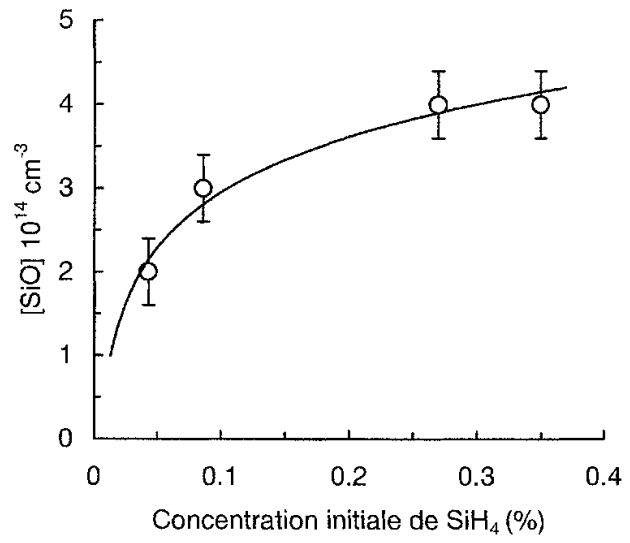

Figure 5. Evolution temporelle de [SiO] fonction de la concentration initiale de silane, déduite de mesures d'autoabsorption - Tension appliquée $=18 \mathrm{kV}$

\section{CONCLUSION}

Pour des mesures de concentrations basées sur le principe de l'absorption, il est nécessaire de bien caractériser le rayonnement incident (auto-absorbé ou non) émis par le réacteur sonde, afin de construire un spectre synthétique absorbé le plus proche possible du spectre expérimental.

La caractérisation de spectres auto-absorbés peut aussi représenter un diagnostic de mesure de concentration d'espèces. Par contre ce type de diagnostic présente deux limites essentielles, d'une part la fluorescence n'est accessible que durant la phase de fluorescence et d'autre part la sensibilité de la méthode d'auto-absorption est limitée par le produit [SiO] $\times$ (reactor length) $>10^{15} \mathrm{~cm}^{-2}$.

Ces techniques nous ont permis d'observer $\mathrm{SiO}$ en grande quantité $\left(\approx 10^{14} \mathrm{~cm}^{-3}\right)$ tard dans la post-décharge (plusieurs millisecondes)

En raison du caractère non-homogène des plasmas de DBD, il faut considérer les estimations de concentration comme des valeurs moyennées sur la longueur du réacteur.

\section{Remerciements}

Les auteurs remercient la société L'Air Liquide pour son support financier.

\section{References}

[1] Van De Weijer P. and Zwerver B. H. Chem Phys Letters 163(1989) 48

[2] Matsuo Y., Nakajima T.,Kobayashi T. and Takami M. Appl. Phys. Lett. 71(1997) 996

[3] Amorim J., Baravian G. and Jolly J., J. Phys. D: Appl. Phys. 33(2000) 51

[4] Kovacs I., "Rotational structure in the spectrum of diatomic molecules", Akademiai Kiado, Budapest 1969

[5] Park C.S., Crosley D. R., Eckstrom D. J. and Heere K. R., J. Quant. Spectrosc. Radiat. Transfer 49(1993) 349

[6] Field R. W., Lagerquist A. and Renhorn I., Physica Scripta 14(1976) 298

[7] Hermann J., Coursimault F., Motret O., Acquaviva S. and Perrone A., J. Phys. B : At. Mol. Opt. Phys. 34(2001) 1917 
Génération de rayonnement $X$, imagerie et applications 